\title{
Discrete Maths Summer in Slovenia
}

Nino Bašić (University of Primorska, Koper, Slovenia), Ademir Hujdurović (University of Primorska, Koper, Slovenia), Matjaž Konvalinka (University of Ljubljana, Slovenia) and Klavdija Kutnar (University of Primorska, Koper, Slovenia)

This summer, mathematical activities in Slovenia will be marked by discrete maths. Some basic information regarding four events are presented in subsequent sections: Maps $\cap$ Configurations $\cap$ Polytopes $\cap$ Molecules $\subseteq$ Graphs: The mathematics of Tomaž Pisanski on the occasion of his 70th birthday, Ljubljana, May 23-25; the 9th Slovenian International Conference on Graph Theory, Lake Bled, June 23-29; the 31st International Conference on Formal Power Series and Algebraic Combinatorics, Ljubljana, July 1-5; and the 9th PhD Summer School in Discrete Mathematics, Rogla, June 30-July 6.

\section{Bled'19}

Bled'19 is the 9th edition of the quadrennial Slovenian Graph Theory Conference. The conference has progressed a long way since its inaugural edition in 1985 (held in Dubrovnik, Croatia). The number of participants has grown from just 30 in 1985 to over 300 at the 8th edition held in Kranjska Gora in 2015, representing all six continents. It has become one of the largest - and for many areas of graph theory, the premier - graph theory conference series, and is attended by leading researchers in graph theory, as well as many postdocs and talented $\mathrm{PhD}$ students. For Bled'19, the number of participants is expected to be comparable to that of 2015, with over 220 talks. In addition to the keynote speakers named below, the conference will consist of minisymposia from specific fields, ranging from algebraic, algorithmic, geometric, topological and other aspects of graph theory to a general session and poster session.

The conference is organized by IMFM - Institute of Mathematics, Physics and Mechanics, Ljubljana, in collaboration with SDAMS (Slovenian Discrete and Applied Mathematics Society), UL FMF (University of Ljubljana, Faculty of Mathematics and Physics), UM FNM (University of Maribor, Faculty of Natural Sciences and Mathematics), UP FAMNIT (University of Primorska, Faculty of Mathematics, Natural Sciences and

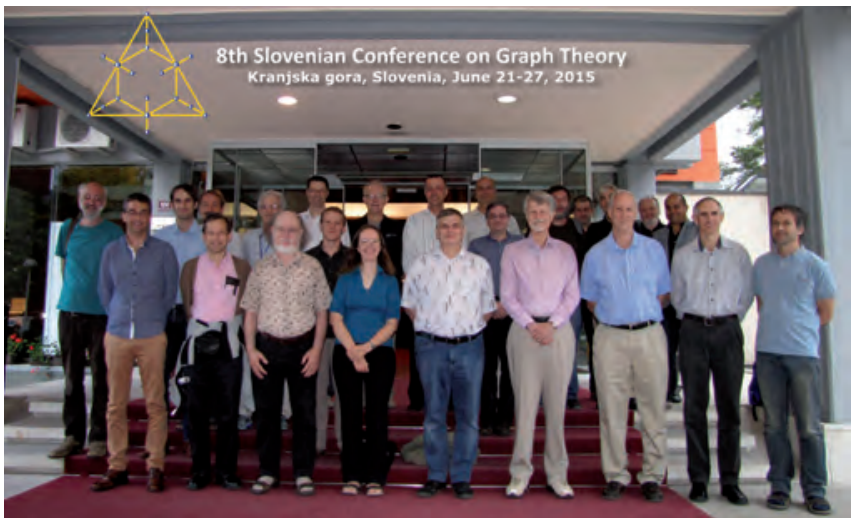

Plenary and invited speakers together with organizers of the 8th Slovenian Conference on Graph Theory.
Information Technologies), and UP IAM (University of Primorska, Andrej Marušič Institute).

\section{List of keynote speakers:}

Noga Alon (Princeton University, USA, and Tel Aviv University, Israel)

Marco Buratti (University of Perugia, Italy)

Gareth Jones (University of Southampton, UK)

Gábor Korchmáros (University of Basilicata, Italy)

Daniel Král' (Warwick University, UK, and Masaryk University, Czech Republic)

Daniela Kühn (University of Birmingham, UK)

Sergei Lando (National Research University Higher School of Economics, Russia)

János Pach (EPFL, Lausanne and Rényi Institute, Hungary)

Cheryl E. Praeger (University of Western Australia, Australia)

Zsolt Tuza (Rényi Institute and University of Pannonia, Hungary)

Xuding Zhu (Zhejiang Normal University, China)

\section{Scientific Committee:}

Sandi Klavžar, Dragan Marušič, Bojan Mohar (chair), Tomaž Pisanski.

For further information please visit the conference web site: http://conferences.matheo.si/e/bled19.

\section{FPSAC'19}

The International Conference on Formal Power Series and Algebraic Combinatorics is a major annual combinatorial conference that is organised in a different city every year. The 31st edition of FPSAC will take place in Ljubljana, Slovenia. Topics include all aspects of combinatorics and their relations with other areas of mathematics, physics, computer science and biology. The conference will include invited lectures, contributed presentations, poster sessions and software demonstrations. There will be no parallel sessions. The number of attendants is usually between 200 and 250, and we expect a similar number this year.

The conference is organised by the University of Ljubljana, Faculty of Mathematics and Physics in collaboration with UM FNM (University of Maribor, Faculty of Natural Sciences and Mathematics), UP FAMNIT (University of Primorska, Faculty of Mathematics, Natural Sciences and Information Technologies), IMFM (Institute of Mathematics, Physics and Mechanics, Ljubljana) and SDAMS (Slovenian Discrete and Applied Mathematics Society), and is sponsored by a variety of organizations and companies, including the National Science Foundation. 
List of keynote speakers:

Andrej Bauer (University of Ljubljana and IMFM, Slovenia)

Alin Bostan (Inria, France)

Sandra Di Rocco (KTH, Royal Institute of Technology, Sweden)

Eric Katz (Ohio State University, USA)

Caroline Klivans (Brown University, USA)

Nataša Pržulj (University College London, UK)

Vic Reiner (University of Minnesota, USA)

Stephan Wagner (Stellenbosch University, South Africa)

Chuánmíng Zōng (Tianjin University, China)

\section{Program Committee Chairs:}

Sara Billey, Marko Petkovšek, Günter Ziegler.

\section{Organizing Committee Chair:}

Matjaž Konvalinka.

For further information please visit the conference web site: http://fpsac2019.fmf.uni-lj.si/.

\section{9th PhD Summer School in Discrete Mathematics}

The University of Primorska, together with the Slovenian Discrete and Applied Mathematics Society, is organising the 9th PhD Summer School in Discrete Mathematics, which will be held in Rogla, Slovenia, between June the 30th and July the 6th, 2019. This PhD Summer School began in 2011 and has since become an annual event. It is aimed at bringing together $\mathrm{PhD}$ students and postdocs with senior lecturers. The Summer School is truly international, with participants from all over the world. Each year there are around 60 participants, more than half of them students. Financial support is offered for several $\mathrm{PhD}$ students to attend this Summer School, which includes half board accommodation and exemption from payment of the conference fee. Students will have the opportunity to present their results in 15-minute talks, and a three-member committee will decide the winner of the Best Student

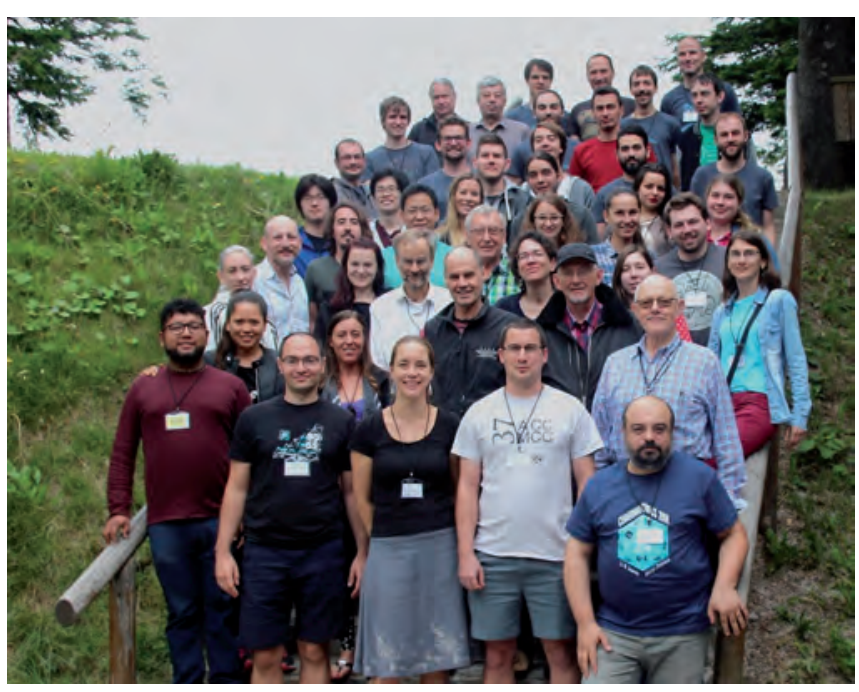

Group photo from the 8th PhD Summer School in Discrete Mathematics.
Talk Award. The winner will receive a certificate and free participation at the 10th PhD Summer School in Discrete Mathematics.

The main part of the summer school consists of the following two mini-courses:

Minicourse 1: Combinatorial limits and their applications in extremal combinatorics, Daniel Král' (Masaryk University, Czech Republic, and University of Warwick, UK)

Minicourse 2: Coxeter groups, Alice Devillers (The University of Western Australia, Perth, Australia).

In addition to the two mini-courses, several invited talks will be given during the summer school.

\section{Confirmed invited speakers are:}

Vida Dujmović (University of Ottawa, Canada)

Miguel Angel Pizana (Universidad Autónoma Metropolitana-Iztapalapa, Mexico)

Jeroen Schillewaert (University of Auckland, New Zealand)

Klara Stokes (Maynooth University, Ireland)

For further information, please visit the conference web site: https://conferences.famnit.upr.si/event/12/.

\section{Scientific Committee:}

Ademir Hujdurović, Klavdija Kutnar, Aleksander Malnič, Dragan Marušič, Štefko Miklavič, Primož Šparl.

\section{Supporters:}

EMS - European Mathematical Society, ARRS - Slovenian Research Agency, MIZŠ - Ministry of Education, Science and Sport RS.

\section{Maps $\cap$ Configurations $\cap$ Polytopes $\cap$ Mol- ecules $\subseteq$ Graphs: The mathematics of Tomaž Pisanski on the occasion of his 70th birthday}

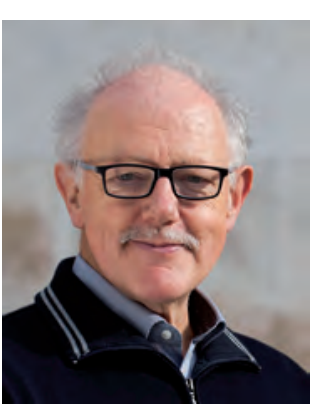

This conference, which was held in Ljubljana, Slovenia, between May the 23rd and 25th, 2019, was organised to celebrate the mathematics of Tomaž Pisanski on the occasion of his 70th birthday. Tomaž Pisanski, known as Tomo to his friends, works in several areas of discrete and computational mathematics. Combinatorial configurations, abstract polytopes, maps on surfaces and chemical graph theory are just a few areas of his broad research interests. Tomo is the author or co-author of over 160 original scientific papers. Together with Brigitte Servatius he authored the book Configurations from a Graphical Viewpoint, which was published in 2013 by Birkhäuser. Tomo's scientific work has been cited over 3400 times according to Google Scholar. In 2008, together with Dragan Marušič, he co-founded Ars 
Mathematica Contemporanea, the first international mathematical journal to be published in Slovenia. Many Slovenian mathematicians refer to Tomo as "the father of Slovenian discrete mathematics". The Mathematics Genealogy Project lists 16 of Tomo's PhD students and 79 academic descendants.

The invited speakers named below are world-class mathematicians who work in areas of mathematics that are close to Tomo. In addition to the invited talks, participants had the opportunity to deliver short, 15-minute presentations.

The conference was organised by UL FMF (University of Ljubljana, Faculty of Mathematics and Physics), in collaboration with UP FAMNIT (University of Primorska, Faculty of Mathematics, Natural Sciences and Information Technologies), UP IAM (University of Primorska, Andrej Marušič Institute), SDAMS (Slovenian Discrete and Applied Mathematics Society), IMFM (Institute of Mathematics, Physics and Mechanics, Ljubljana), and Abelium d.o.o.

List of invited speakers:

Vladimir Batagelj (University of Ljubljana and University of Primorska, Slovenia)

Gunnar Brinkmann (Ghent University, Belgium)

Patrick W. Fowler (The University of Sheffield, United Kingdom)

Gábor Gévay (University of Szeged, Hungary)

Wilfried Imrich (Montanuniversität Leoben, Austria)

Asia Ivić Weiss (York University, Canada)

Sandi Klavžar (University of Ljubljana and University of Maribor, Slovenia)

Dimitri Leemans (Université Libre de Bruxelles, Belgium)

Dragan Marušič (University of Primorska, Slovenia)

Alexander D. Mednykh (Sobolev Institute of Mathematics and Novosibirsk State University, Russian Federation)

Bojan Mohar (Simon Fraser University, Canada, and IMFM, Slovenia)

Daniel Pellicer Covarrubias (National Autonomous University of Mexico, Mexico)

Egon Schulte (Northeastern University, United States)

Brigitte Servatius (Worcester Polytechnic Institute, United States)

Martin Škoviera (Comenius University in Bratislava, Slovakia)

Thomas W. Tucker (Colgate University, United States)

Steve Wilson (Northern Arizona University, United States)

Scientific Committee:

Klavdija Kutnar, Primož Potočnik, Arjana Žitnik.

Organising Committee:

Nino Bašić, Marko Boben, Boris Horvat, Jurij Kovič, Alen Orbanić, Arjana Žitnik.

For further information please visit the conference web site: http://conferences.matheo.si/e/mcpm.

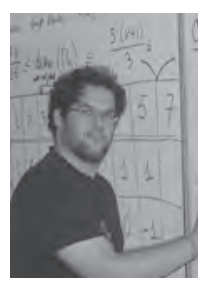

Nino Bašić [nino.basic@upr.si] is an Assistant Professor of Mathematics and Computer Science at the University of Primorska. He is an active member of the SDAMS and a member of the Organising Committee of the $8 E C M$. His research interests include various aspects of graph theory and its applications to natural sciences.

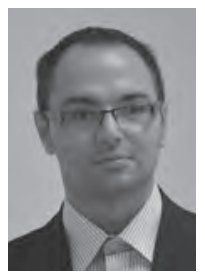

Ademir Hujdurović [ademir.hujdurovic@ upr.si] obtained his PhD degree in mathematics from the University of Primorska, Koper, Slovenia, in 2013. He is currently an Assistant Professor at the University of Primorska and Vice-Dean for International Cooperation of UP FAMNIT. He is also a member of the Organising Committee of the 8ECM. His main research interest is graph theory, mainly in graphs that posses a certain degree of symmetry.

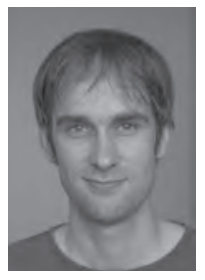

Matjaž Konvalinka [matjaz.konvalinka@ fmf.uni-lj.si] is an Associate Professor of Mathematics at the Faculty of Mathematics and Physics, University of Ljubljana, and the chair of the Organising Committee of FPSAC 2019. His main research interests are algebraic and enumerative combinatorics.

The photo and CV of Klavdija Kutnar can be found in previous Newsletter issues. 